Ophthalmologe $2009 \cdot 106: 52-55$ DOI 10.1007/s00347-008-1810-3

Online publiziert: 11 . September 2008

(c) Springer Medizin Verlag 2008

\author{
A.M. Palmowski-Wolfe ${ }^{1}$ - L. Kappos ${ }^{2}$ - J. Müller-Brand ${ }^{3} \cdot$ C. Buitrago-Tellez ${ }^{4}$. \\ A. Merlo 5 \\ ${ }^{1}$ Augenklinik, Universitätsspital Basel \\ 2 Neurologie, Universitätsspital Basel \\ ${ }^{3}$ Nuklearmedizin, Universitätsspital Basel \\ ${ }^{4}$ Radiologie, Spital Zofingen \\ ${ }^{5}$ Neurochirurgie, Universitätsspital Basel
}

\title{
Belastungsabhängige vertikale Doppelbilder und Ptosis
}

\section{Anamnese}

Eine 43-jährige Patientin wurde uns zugewiesen, weil Drittpersonen auffiel, dass seit 2-3 Monaten ihr linkes Augenlid in wechselnder Ausprägung hing. Subjektiv berichtete die Patientin, dass sie neuerdings auch Doppelbilder in Extrempositionen oder nach schnellen Augenbewegungen wahrnehme. Ferner sei sie lichtempfindlich.

\section{Befund}

Der korrigierte Fernvisus betrug RA: $-1,25=1,0 ;$ LA: $-1,25-0,5 A 63^{\circ}=1,0$.

Die Lidspalte imponierte in Primärposition zunächst symmetrisch, bds. $10 \mathrm{~mm}$, nach Belastung kam es jedoch zu einer zunehmenden Ptose links (• Abb. 1).

Spaltlampenmikroskopisch zeigten sich regelrechte vordere Augenabschnitte, insbesondere Pupillen isokor. Ophthalmoskopie: Papille randscharf, vital, im Niveau, CDR o,1. Makula und periphere Netzhaut waren regelrecht, Gefäße nicht gestaut.

Im Covertest zeigte sich für die Ferne und Nähe eine gut kompensierte Exophorie. Die Stereofunktionen waren gut: Bagolini Ferne/Nähe positiv, ohne Einstellbewegung. TNO bis 60 " positiv.

Bei Prüfung der Motilität zeigte sich am linken Auge eine leichte Einschränkung der Hebung und der Adduktion
(- Abb. 2), wobei die Hebung bei zunehmendem Aufblick abnahm. Dementsprechend äußerte die Patientin vertikale Doppelbilder im Aufblick, welche nach längerer Blickhaltung zunahmen. In der Harms-Wand wurden auch Doppelbilder im extremen Seitblick sowie in starkem Abblick angegeben (• Abb. 3).

\section{Weitere Diagnostik}

Bei belastungsabhängigen Beschwerden wurden zunächst im Hinblick auf eine mögliche Myasthenie Acetylcholinrezeptorantikörper bestimmt, die jedoch ebenfalls wie AK gegen quergestreifte Muskulatur im Normbereich waren. Eine repetitive Stimulation zeigte kein pathologisches Dekrement. Im Tensilontest trat keine Besserung ein.

Im MRI zeigte sich eine Raumforderung im Sinus cavernosus links. Diese ummauerte die A. carotis interna und stenosiert sie um ca 1/3 (• Abb. 4). Mittels einer ${ }^{111}$ In-Octreotide-Scintigraphie (Octreoscan $^{\oplus}$ ) konnte bewiesen werden, dass der Tumor Somatostatinrezeptoren in hohem Ausmaß exprimiert. Somit wurde die Diagnose eines Keilbeinmeningeoms gestützt. 

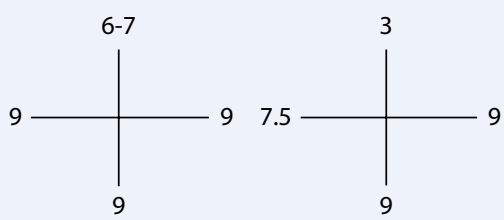

Abb. $2 \Delta$ Ein Vergleich der monokularen Exkursionen des rechten Auges (links) mit denen des linken Auges (rechts), gemessen in mm nach Kestenbaum (Limbustest), zeigt links eine messbar verminderte Hebung und Adduktion
Abb. $1 \triangleright$ Der untere Teil zeigt, im Vergleich zum oberen, die zunehmende Ptose des linken Auges nach längerem Aufblick
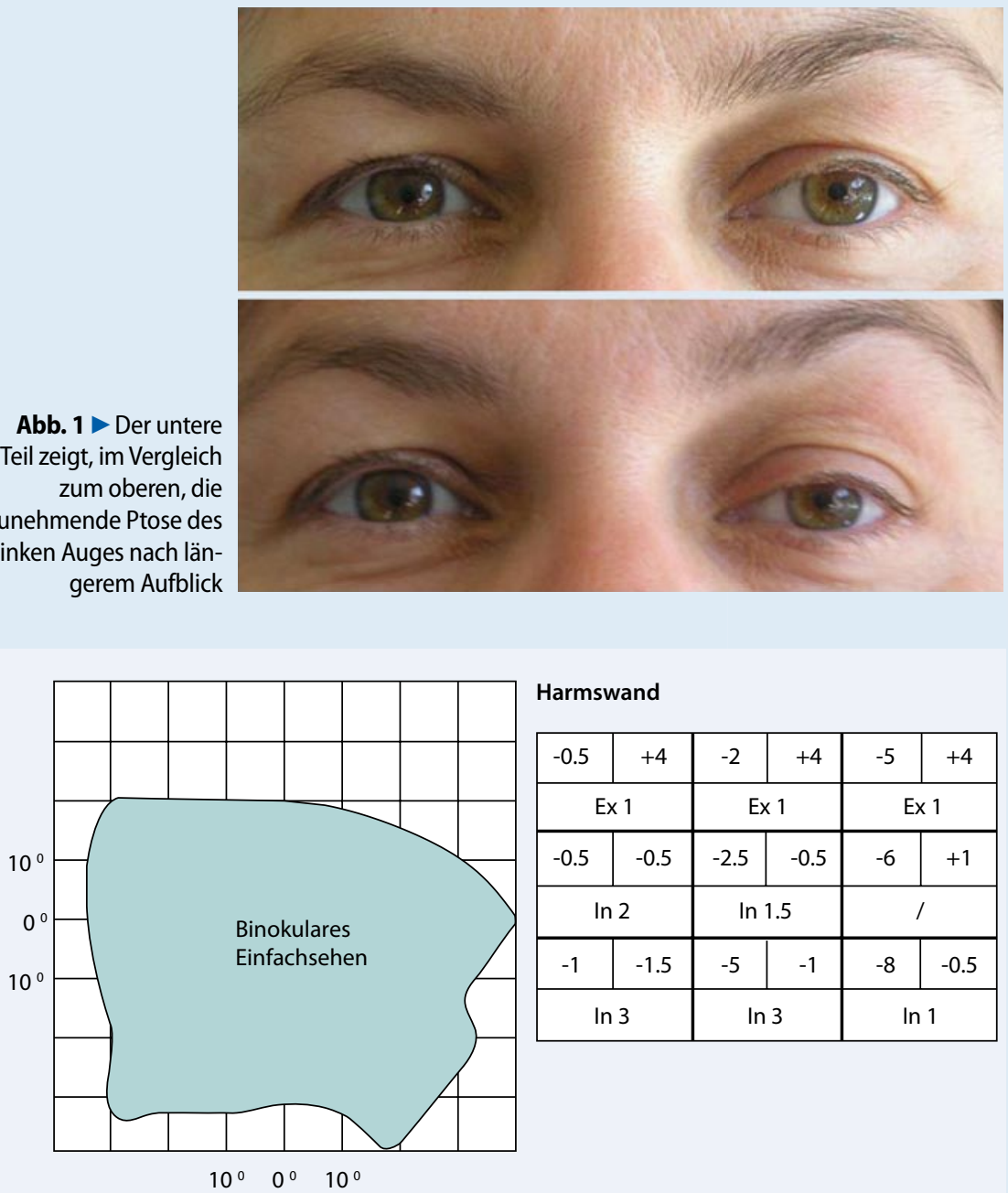

Abb. $3 \Delta$ Diese Abbildung zeigt das Feld des binokularen Einfachsehens vor der Behandlung (blau). An der Harms-Wand sind für die 9 Blickrichtungen die Werte (gemessen in Grad) der seitlichen Abweichung der Augen links, der Höhenabweichung rechts und der rotatorischen Abweichung (darunter) gezeigt

Abb. $4 \triangleright$ Im MRI zeigt sich eine Raumforderung im linken Sinus cavernosus. Dieser ummauert die $A$. carotis interna

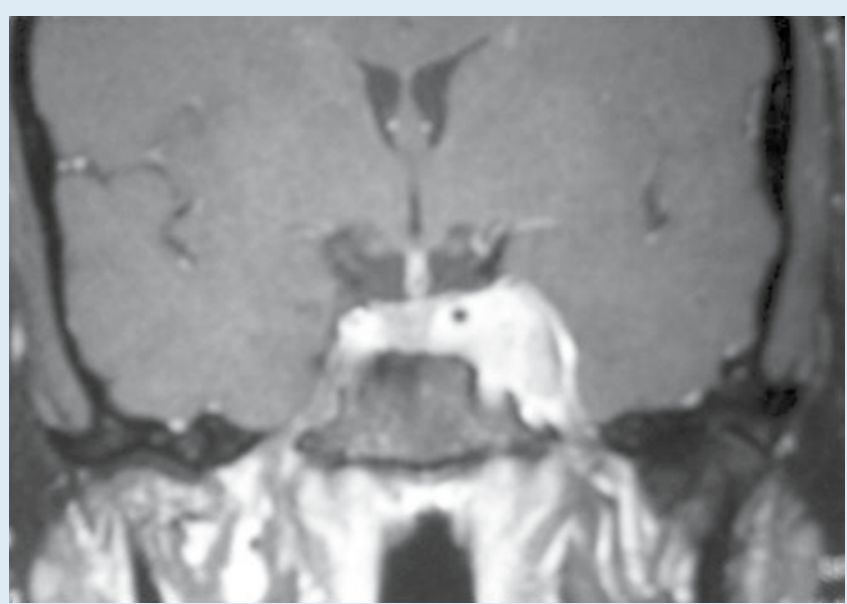




\section{> Diagnose: Okuläre Pseudomyasthenie bei Keilbeinmeningeom am Sinus cavernosus links}

\section{Therapie und Verlauf}

Das Keilbeinmeningeom wurde in der Folge 2-mal innerhalb von 3 Monaten mittels Radiopeptidtherapie mit ${ }^{\circ}$ Y-DOTATOC (Somatostatin-Analogon) behandelt [2]. Hierunter kam es zur Größenregredienz des Tumors mit einer Besserung der klinischen Symptomatik. Die Patientin ist nun seit mehr als 3 Jahren stabil. Eine Lidasymmetrie bemerkt sie nicht mehr. Sie meidet im Alltag extreme Blickrichtungen und ist mit dieser Strategie beschwerdefrei, zudem sie für die Nähe 1-Prisma-Basis oben LA benützt.

\section{Diskussion}

Hirntumoren können selten über fluktuierende motorische Funktionsstörungen, die z. T. auch auf Cholinesterasehemmer ansprechen, eine Myasthenie vortäuschen [5]. In der Literatur wurde die sog. okuläre Pseudomyasthenie vereinzelt bei Patienten mit parasellären intrakraniellen Tumoren beschrieben [4]. Daher sollte bei Patienten mit belastungsabhängigen Beschwerden, die an eine Myasthenie erinnern, bei unzureichend gesicherter Myasthenie immer auch eine Bildgebung zum Ausschluss eines intrakraniellen Tumors erfolgen. Der Sinus cavernosus ist vom Keilbein (Os sphenoidale) und dem Os temporale begrenzt. Er beinhaltet ein Venengeflecht, die A. carotis interna mit dem umgebenden Plexus sympathicus sowie die Hirnnerven III, IV, $V_{1}, V_{2}$, VI. Dementsprechend können Raumforderungen die Hirnnerven durch Druck oder möglicherweise durch Induktion einer Minderperfusion schädigen. Eine blickrichtungsabhängige Amaurose wurde bei einem $\mathrm{Pa}$ tienten mit einem Meningeom im Sinus cavernosus beschrieben. Ein vaskulärer Pathomechanismus wurde postuliert, zumal bei entsprechender Blickrichtung, mittels Farbdoppler, ein höherer Widerstandsindex in der A. centralis retina ge- messen werden konnte. Möglicherweise bewirkten Kompression, Distorsion und oder Verlagerung der A. carotis interna durch das Meningeom eine Minderperfusion, die blickrichtungsabhängig durch Zugwirkung verstärkt wurde [3]. Auch bei unserer Patientin ist ein ähnlicher $\mathrm{Pa}$ thomechanismus denkbar: Die Befunde (Ptosis, Hebungs- und Adduktionseinschränkung) sind mit einer partiellen Beeinträchtigung des N. oculomotorius vereinbar. Das Keilbeinmeningeom kann zum einen direkt auf den N. III und die Vasa nervorum drücken und so nicht nur eine Kompression der Arteriolen bewirken, sondern auch eine venöse Hyperämie hervorrufen. In einer anatomischen Studie konnte gezeigt werden, dass der N. III im Bereich des Sinus cavernosus über Arteriolen aus dem kavernösen Anteil der A. carotis interna versorgt wird [1]. Diese Arterie ist bei unserer Patientin linksseitig ummauert und hochgradig eingeengt, sodass eine Minderperfusion auch der abgehenden Gefäße in diesem Bereich wahrscheinlich erscheint. Bei Belastung reicht dann die Perfusion nicht mehr aus, um dem gesteigerten Energiebedarf gerecht zu werden.

Ein chirurgisches Vorgehen birgt im Sinus cavernosus ein hohes Risiko der Morbidität und sogar ein 5\%-Mortalitätsrisiko [6]. Bei unserer Patientin konnten Octreotidrezeptoren auf dem Tumor nachgewiesen werden. Bei solchen Raumforderungen bietet sich der Versuch einer metabolischen Therapie an. Bei der Behandlung mit ${ }^{\circ}$ Y-DOTATOC wird ein Betastrahler, Yttrium, mit einem Octreotidderivat gekoppelt venös injiziert [2]. Die radioaktive Substanz reichert sich an den Octreotidrezeptoren an und entfaltet ihre Hauptwirkung in einem Radius von $1 \mathrm{~cm}$. Die HWZ ist mit 2,7 Tagen recht kurz. Bei unserer Patientin führte diese Therapie zu einer Volumenreduktion des Tumors sowie zu einer klinischen Verbesserung und dann Stabilisierung.

\section{Korrespondenzadresse \\ Prof. Dr. A.M. Palmowski-Wolfe

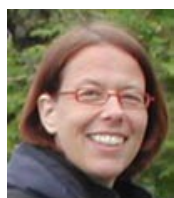 \\ Augenklinik, Universitäts- spital Basel Mittlere Straße 91, 4031 Basel Schweiz \\ PalmowskiA@uhbs.ch}

Interessenkonflikt. Der korrespondierende Autor gibt an, dass kein Interessenkonflikt besteht.

\section{Literatur}

1. Cahill M, Bannigan J, Eustace P (1996) Anatomy of the extraneural blood supply to the intracranial oculomotor nerve. Br J Ophthalmol 80: 177-181

2. Forrer F, Waldherr C, Maecke HR et al. (2006) Targeted radionuclide therapy with $90 \mathrm{Y}-\mathrm{DOTATOC}$ in patients with neuroendocrine tumors. Anticancer Res 26: 703-707

3. Koch MU, Houtman AC, Dekeizer R (2006) Gazeevoked Amaurosis with cavernous sinus meningeoma. Eye 20: 840-866

4. Moorthy G, Behrens MM, Drachman DB et al. (1989) Ocular pseudomyasthenia or ocular myasthenia, plus': a warning to clinicians. Neurology 39 : 1150-1154

5. Rodolico C, Girlanda P, Nicolosi C et al. (2003) Chiari I malformation mimicking myasthenia gravis J Neurol Neurosurg Psychiatry 74: 393

6. Sindou M, Wydh E, Jouanneau E et al. (2007) Longterm follow-up of meningiomas of the cavernous sinus after surgical treatment alone. J Neurosurg 107: 937-944 
Hier steht eine Anzeige.

算 Springer 